\title{
Possible ameliorative effects of pentoxifylline on cisplatin-induced ototoxicity in rats: a study with hearing test, light, and scanning electron microscopy
}

\author{
Marwa A. Al-Gholam ${ }^{1}$, Asmaa S. Moaty ${ }^{2}$, Ahmed M. Zein-Elabedein ${ }^{2}$, Asmaa S. Essawy ${ }^{1,3}$ \\ ${ }^{1}$ Human Anatomy and Embryology Department, Faculty of Medicine, Menoufia University, Menoufia, Egypt \\ ${ }^{2}$ Audiovestibular unit, Otolaryngology Department, Faculty of Medicine, Menoufia University, Menoufia, Egypt \\ ${ }^{3}$ Anatomy Department, Ibn Sina National College for Medical Studies, Jeddah, Saudi Arabia
}

\section{SUMMARY}

Cisplatin is an antineoplastic drug widely used to treat various types of cancer. Ototoxicity is still cisplatin's most critical side-effect. Some patients may experience dose limitations due to hearing loss. Pentoxifylline (PX) exhibits powerful antioxidant, anti-inflammatory, and immuneregulatory effects. Our study was designed to investigate the protective effects of pentoxifylline on cisplatin-induced ototoxicity. Forty adult male healthy Sprague-Dawley rats were used through the entire experiment. Four groups of animals were categorized: Group I (control group); Group II (PX group) received $25 \mathrm{mg} / \mathrm{kg} /$ day of oral PX by gavage for 8 consecutive days; Group III (Cisplatin group) received cisplatin single intraperitoneal dose of $10 \mathrm{mg} / \mathrm{kg}$; Group IV (PX + Cisplatin group) received $25 \mathrm{mg} / \mathrm{kg} /$ day of oral PX by gavage for 8 successive days and a single intraperitoneal dose of $10 \mathrm{mg} / \mathrm{kg}$ cisplatin at the 4th day. First and $9^{\text {th }}$-day Distortiondistortionproduct otoacoustic emissions (DPOAE) tests were conducted. An intracardiac blood sample was collected for total antioxidant capacity (TAC) measurement, and the cochleae of rats were examined histopathologically. A significant reduction in serum TAC value was detected in the cisplatin group, while PX treatment significantly reduced TAC. Cisplatin decreased the DPOAE amplitudes in rats; conversely, the PX+cisplatin group showed a significant increase at all frequencies. Upon histopathological examination, the Ciplastin group revealed perturbation of the normal architecture of the organ of Corti, increased collagen deposition and marked expression of caspase- 3 , while the PX+cisplatin group revealed preserved architecture of the organ of Corti, minimal collagen deposition and downregulation of Caspase-3 expression. As evidenced by our findings and results from DPOAE results, biochemical findings, histological and ultrastructural analyses, PX significantly protects rats against ototoxicity caused by cisplatin.

Key words: Pentoxifylline - Cisplatin Ototoxicity - Rats 


\section{INTRODUCTION}

The term ototoxicity alludes to cellular damage or functional disorders that develop in the inner ear associated with any therapeutic or chemical (Kara et al., 2016). Cisplatin (cisdiamminedichloroplatin II) is widely used for treatment of a number of tumors (testicular and ovarian carcinoma, squamous head and neck carcinoma, lung carcinoma, malignant gliomas, advanced bladder cancer, and metastatic cancers such as mesothelioma, melanoma, breast and prostate cancer). The treatment with cisplatin is associated with side effects, such as ototoxicity, nephrotoxicity, myelotoxicity, and toxicity to the gastrointestinal system. Even though hydration therapy can control nephrotoxicity, ototoxicity remains a serious concern with cancer treatment with cisplatin (Roldan-Fidalgo et al., 2016).

Bilateral, irreversible, progressive sensorineural loss of hearing at high frequencies as well as tinnitus are the hallmarks of ototoxicity caused by cisplatin. Some patients may experience dose limitations due to hearing loss. The outer hair cells of the cochlea are progressively damaged by Cisplatin. 60-80\% of patients who receive cisplatin suffer bilateral, permanent, and progressive hearing loss (Salehi et al., 2014). The severity of deafness can vary greatly, and it does not pose a life-threatening challenge. However, hearing loss can significantly affect the quality of life and can result in communication problems. Molecular and cellular mechanisms determining cisplatin toxicity remain poorly understood and the reduction of cisplatin ototoxicity remains one of the major objectives of anti-tumor therapies (Yumusakhuylu et al., 2012).

Cisplatin ototoxicity is associated with the accumulation of reactive oxygen species (ROS), including hydroxyl radicals and superoxide ions, and inhibition of antioxidant enzymes; therefore, antioxidants are popular agents to mitigate its toxicity (Rybak, 2007). Exogenous antioxidants such as amifostine, erdosteine, lycopene, vitamins $\mathrm{C}$ and $\mathrm{E}$, dexamethasone, sodium salicylate, aminoguanidine, methionine, and resveratrol have been shown to have protective benefits in several studies (Kalkanis et al., 2004; Erdem et al., 2012; Xie et al., 2011).
Pentoxifylline (PX) is an alkaloid created by adding a hexanone group to theobromine, which is extracted from cocoa beans (Gill et al., 2015). As a nonspecific phosphodiesterase inhibitor, it was originally considered to treat peripheral vascular diseases. PX has several pharmacologic effects, such as reducing blood viscosity, improving microcirculation, increasing erythrocyte deformability, inhibiting platelets from aggregating in addition to its anti-inflammatory, immunomodulatory and antiproliferative properties (Nasiri-Toosi et al., 2013). Furthermore, it has also been used as an antioxidant to heal various tissue damages (Stojiljkovic et al., 2009). Considering oxidants' role in cisplatin-induced ototoxicity and the useful properties of pentoxifylline, this study has evaluated the protective effects of pentoxifylline against cisplatin-induced ototoxicity and the potential mechanisms through which it works.

\section{MATERIALS AND METHODS}

\section{Chemicals}

Pentoxifylline (PX) used in the study was obtained in the form of film-coated tablets (Trental) produced by Sanofi-Aventis, Egypt under licence of Sanofi-Aventis, Germany, and cisplatin as solution vials was obtained from Mylan, Greece (1 mg/ml).

\section{Experimental design}

This study was performed at the department of Human Anatomy and Embryology, Faculty of Medicine, Menoufia University. Forty adult male Sprague-Dawley rats weighing 180-200 g, aged 10-12 weeks, were used in the study. Rats were bought from the Egyptian Organization for Biological Products and Vaccines (Cairo, Egypt). They were acclimatized for two weeks in stainless steel cages prior to starting the experiment. The rats were fed ad libitum on standard laboratory pellets and with tap water. The room was kept at an optimum temperature and humidity with a 12hour light/12-hour dark cycle.

All experimental procedures and animal maintenance were carried out according to the guidelines and roles prescribed by the Research 
Ethics Committee, Menoufia University, Faculty of Medicine with the ethical approval number 7/2020ANAT2.

After two weeks of acclimatization, animals were separated randomly into four groups $(n=10$ per group):

- Group I (Control group): was subdivided into:

GIa (plain control), where animals did not receive anything.

GIb (sham control), where animals received $1 \mathrm{ml}$ intraperitoneal saline solution/day for eight successive days.

- Group II (PX group): each rat received $25 \mathrm{mg} /$ $\mathrm{kg} /$ day of oral pentoxifylline dissolved in 1 $\mathrm{ml}$ saline via gastric gavage for 8 consecutive days (El-Anwar et al., 2018).

- Group III (Cisplatin group): each rat received a single cisplatin intraperitoneal dose of $10 \mathrm{mg} /$ $\mathrm{kg}$ on the $4^{\text {th }}$ day of the experiment (De Araujo et al., 2019).

- Group IV (PX + Cisplatin group): Each rat received $25 \mathrm{mg} / \mathrm{kg} /$ day of oral pentoxifylline dissolved in $1 \mathrm{ml}$ saline via gastric gavage for 8 consecutive days and a single intraperitoneal dose of $10 \mathrm{mg} / \mathrm{kg}$ cisplatin at the $4^{\text {th }}$ day (De Araujo et al., 2019).

An otoscopic examination was performed on all rats prior to the delivery of all medications under $50 \mathrm{mg} / \mathrm{kg}$ ketamine hydrochloride and 5 $\mathrm{mg} / \mathrm{kg}$ xylazine anesthesia according to Struck et al. (2011). Then, all rats were subjected to the Distortion Product Otoacoustic Emissions (DPOAE) test. Under the same anesthesia protocol, DPOAE was again performed on the 9th day of the study. Rats were anesthetized 24 hours after the end of the experiment. A sample of intracardiac blood was taken utilizing plain tubes from each animal following completion of the final auditory assessment. After blood collection, all rats were sacrificed, and the temporal bones were then dissected. The cochleae were extracted, and cochleae from the right ear were prepared for light microscopic examinatio,n while those from the left ear were enucleated and processed for scanning electron microscopy.

\section{Biochemical study for measurement of total an- tioxidant capacity (TAC)}

After collecting blood samples and centrifuging them at $3000 \mathrm{rpm}$ for $15 \mathrm{~min}$, serum was then collected and stored at $80^{\circ} \mathrm{C}$ until being analyzed. Total antioxidant capacity (TAC) (Biodiagnostic, CAT. NO TA2513, Egypt) was measured colorimetrically, according to Koracevic et al. (2001). Serum TAC was assessed by the reaction of antioxidants in the sample with a known amount of exogenously provided hydrogen peroxide. TAC is measured in (mM/l) for each plasma sample.

\section{Otoacoustic Emission Measurements}

Distortion-product otoacoustic emissions (DPOAE) levels were assessed on the first day of the study as a baseline assessment using Eclipse 25 device (Eclipse/EP25 system, Inter acoustics Inc., Middle fart, Denmark). The rats were anesthetized before the assessment and submitted to otoscopic examination. The OAE probe with neonatal ear tips was inserted in the external auditory canal of the ear of the animals; animals with abnormal DPOAE measurements denoting abnormal hearing were excluded from the study. The resulting otoacoustic emissions levels were assessed at 2, 4, 6, and $8 \mathrm{kHz}$ through DP gram. 2 frequencies were used for assessment: $\mathrm{F} 1$ and $\mathrm{F} 2$ with $\mathrm{F} 2 / \mathrm{F} 1$ ratio $=1.22$ at $65 \mathrm{~dB}$ SPL, and the DPOAE levels were assessed at the first day (D1) and day 9 (D9) after administration of the otoprotective agent.

\section{Light microscopic examination}

On the apex of the right cochlea, a small hole was induced. After 2 days of incubation in 10\% neutral buffered formalin, the specimens were put into ethylenediaminetetraacetic acid (EDTA) at $4{ }^{\circ} \mathrm{C}$ for 20 days; the solution was changed every two days until softening occurred. Washing, dehydrating, clearing, and embedding in paraffin blocks were performed. The sections were cut at five- $\mu \mathrm{m}$ and stained with Hematoxylin and Eosin (H\&E) and Masson Trichrome stains. Further deparaffinizing of the paraffinembedded sections was performed in xylene, and rehydrating with graded concentrations of ethanol to be immunohistochemically stained 
with primary anti-Caspase- 3 antibodies, a marker for apoptosis (rabbit polyclonal, Abcam), with a concentration of $100 \mu \mathrm{l}$ at $0.872 \mathrm{mg} / \mathrm{ml}$ (dilution 1/50). The primary antibodies were applied on the slides and incubated overnight at room temperature in humidity chamber. All procedures were performed following the instructions of manufacturer (Suvarna et al., 2012).

\section{Scanning electron microscopic examination}

A small hole has been made on the apex of the left cochlea, washed repeatedly using PBS, and carefully dissected under a dissection microscope (Olympus SZ×9) to reveal the organ of Corti. Following dehydration in graded ethanol series, the specimens were critical-point dried using a CP dryer (Balzers, Lichtenstein). They were attached to aluminum stubs using carbon glue (Planocarbon, Groepl, Austria), coated with a 10-15-nm layer of gold-palladium in a Baltech MED 020 coating system, and examined using a scanning electron microscope (JSM- 6510 LV) (JEOL, Japan) at the Electron MicroscopeU in the Faculty of Agriculture, EL-Mansoura University, ElMansoura, Egypt. To understand the morphology of hair cells, several photomicrographs at different magnifications were taken (Amora et al., 2013).

\section{Statistical analysis}

SPSS (statistical package for social science) version 23.0 on an IBM compatible computer (SPSS Inc., Chicago, IL, USA) was used to analyze the data. For comparisons of the studied groups, we used one-way-ANOVA followed by post hoc Bonferroni tests. DPOAE values were compared before and after drug application in each group using Paired samples t-tests. Data were expressed as the mean \pm standard deviation (SD). P-values less than 0.05 were considered statistically significant, whereas those more than 0.05 were declared non-significant.

\section{RESULTS}

\section{Total antioxidant capacity results}

Compared with the control group, cisplatin significantly reduced serum TAC $(\mathrm{mM} / \mathrm{l}) \quad(P<$ 0.001). In comparison with cisplatin, $P X$ treatment significantly reduced serum TAC levels $(P<0.01)$ (Fig. 1).

\section{DPOAE results}

DPOAE values did not differ significantly in terms of amplitude between the right and left ears of the rats. In the rats of the cisplatin group, a significant decrease in DPOAE amplitude

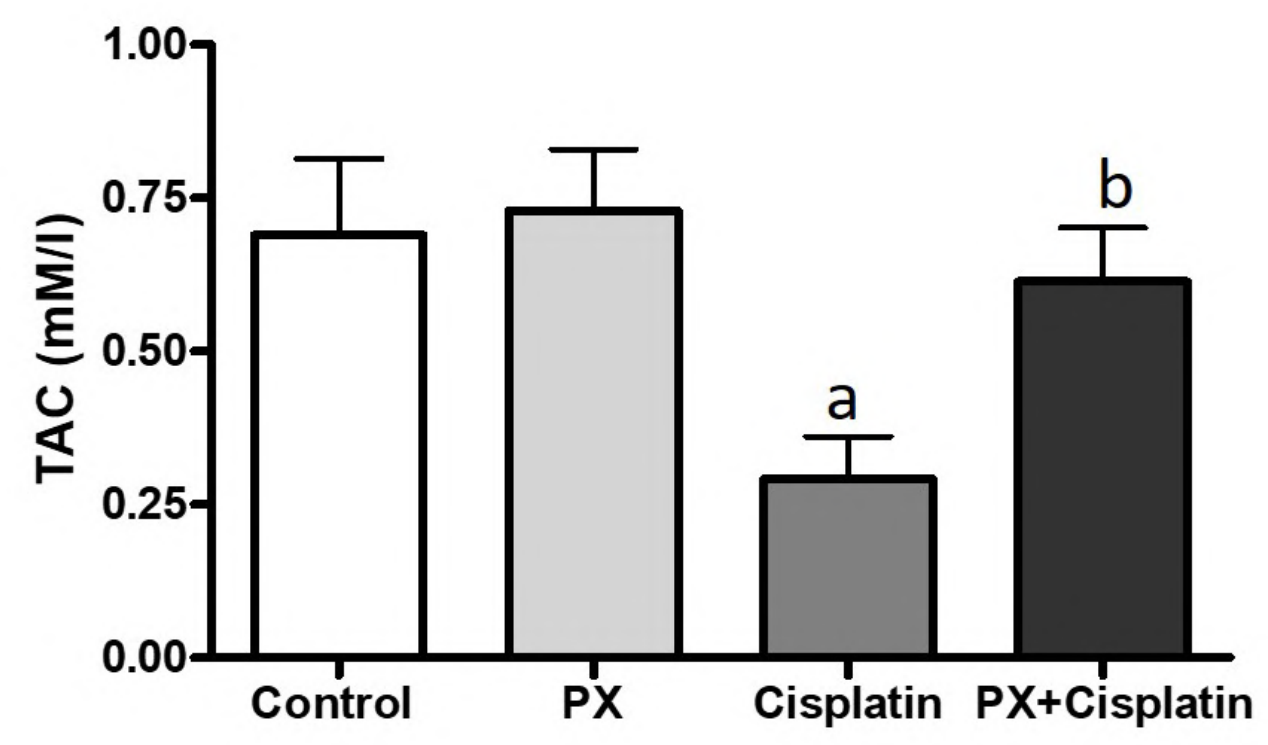

Fig. 1.- Total antioxidant capacity (TAC) results of different experimental groups. a, $\mathrm{P}<0.001$ in comparison with the control group. b, $\mathrm{P}<0.001$ based on cisplatin group comparison. 
was observed on day 9 compared to day 1 at all frequencies $(p<0.001)$. DPOAE values did not differ significantly in terms of amplitude between control, PX and PX+cisplatin Groups compared to day 1 values. At all frequencies, there was marked decline in DPOAE amplitudes in the cisplatin group in comparison to the control group comparing the DPOAE amplitudes on day 9 ( $\mathrm{p}<0.001)$. Comparatively to the cisplatin group, the $\mathrm{PX}+$ cisplatin group exhibited greater DPOAE amplitudes values at all frequencies $(\mathrm{p}<$ 0.001) (Fig. 2).

\section{Histopathological results}

Control and PX groups had similar results for all the examined parameters. Therefore, they are regarded both as controls. On H\&E-stained cochlear sections from the control group, the organ of Corti, stria vascularis, and spiral ganglion were visible. Among the organ of Corti's features are a spiral limbus, homogeneous tectorial membrane, three columnar outer hair cells, one columnar inner hair cell, outer and inner phalangeal cells, and outer and inner pillar cells (Fig. 3A). The stria vascularis was found to consist of three layers: a dark superficial marginal layer, a light intermediate layer, and a light basal layer attached to the spiral ligament (Fig. 3D). There were large cell bodies of pseudo-unipolar neurons with prominent central rounded nuclei enclosed in a capsule of satellite cells in spiral ganglia (Fig. 3G).

In the cisplatin group, the normal architecture of the organ of Corti was disrupted. All the hair cells, as well as their supporting cells, were fragmented and destroyed with a disrupted basement membrane (Fig. 3B). Vacuolation and degeneration of Stria vascularis marginal cells, the apparent increase in thickness, and vacuolation of CT underneath stria vascularis were detected (Fig. $3 \mathrm{E})$. Vacuolation, hemorrhage and degeneration of the nuclei of the spiral ganglia were noted (Fig. $3 \mathrm{H})$. The PX+cisplatin group showed preserved tectorial membrane architectures, intact outer and inner hair cells, and other supporting cells (Fig. 3C). Stria vascularis displayed intact layers with vacuolation of the underlying CT (Fig. 3F). Few vacuolated cells were present in the intact spiral ganglion neurons (Fig. 3I).

Minimal collagen fibers deposition in the basilar membrane and the area underlying stria vascularis in the control and PX+cisplatin groups. However, the cisplatin group shows increased collagen deposition (Fig. 4).

\section{Immunohistochemical results}

A faint brown reaction was detected in the organ of Corti, stria vascularis, and spiral ganglia in the

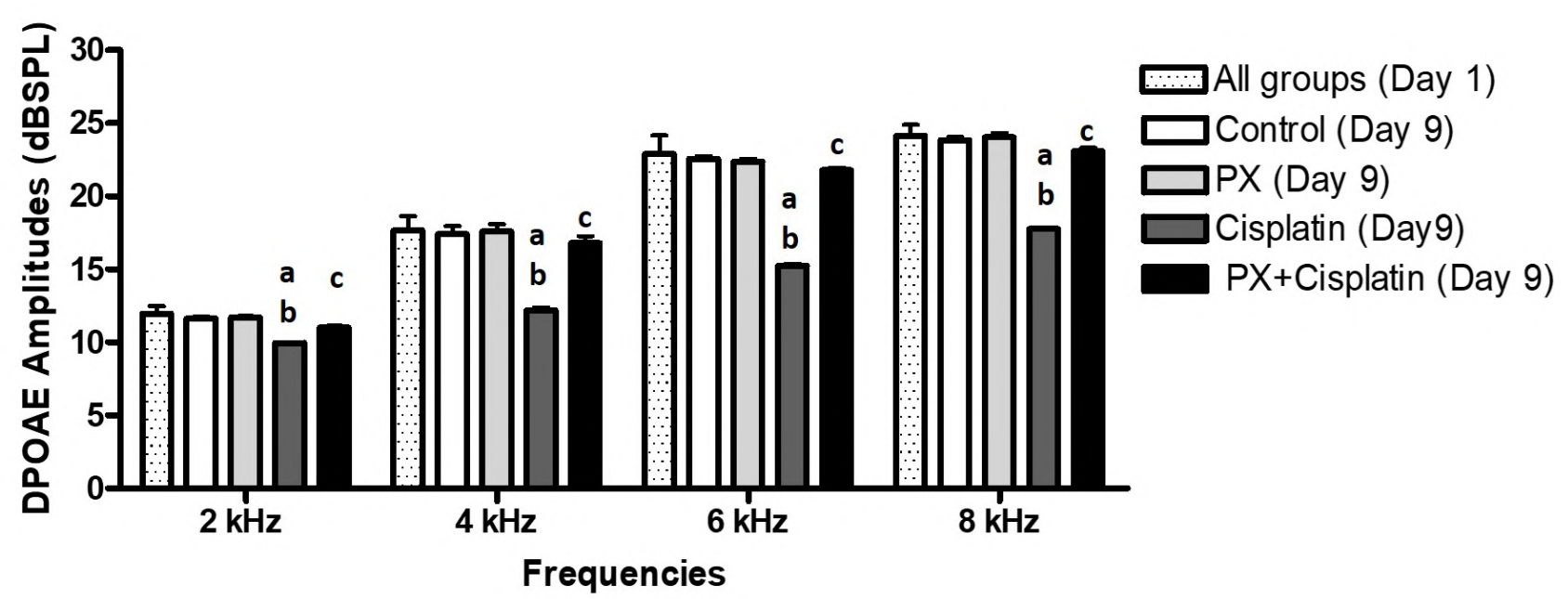

Fig. 2.- DPOAE results of different experimental groups. a, $\mathrm{P}<0.001$ when compared to day 1 . b, $\mathrm{P}<0.001$ comparatively to the control group. $\mathrm{c}, \mathrm{P}<0.001$ comparatively to the cisplatin group. 
control group when caspase-3 immunostained sections were examined (Fig. $5 \mathrm{~A}, \mathrm{D}, \mathrm{G}$ ). In contrast, the cisplatin group showed marked expression in the organ of Corti, stria vascularis and spiral ganglia (Fig. 5 B, E, H). PX treatment showed downregulation of Caspase-3 expression (Fig. 5 C, F, I).

\section{Scanning electron microscopic results}

The control group cochlea was revealed to be a spiral tunnel by SEM. Three and a half turns could be seen in the tunnel from its base to its apex
(Fig. 6A). A cast of cochlear normal vasculature was observed (Fig. 6B). The stereocilia on OHCs appeared to be arranged in three V-shaped rows, while the IHCs were arranged in a linear row. Both of them were regular, erect and well organized (Fig. 7 A, B, C).

Cochleae of cisplatin group showed collapsed cast of blood vessels (Fig. 6C). Stereocilia of outer and inner hair cells were destroyed, fragmented, merged, and disfigured;. cavitation was also revealed (Fig. 7 D, E, F).

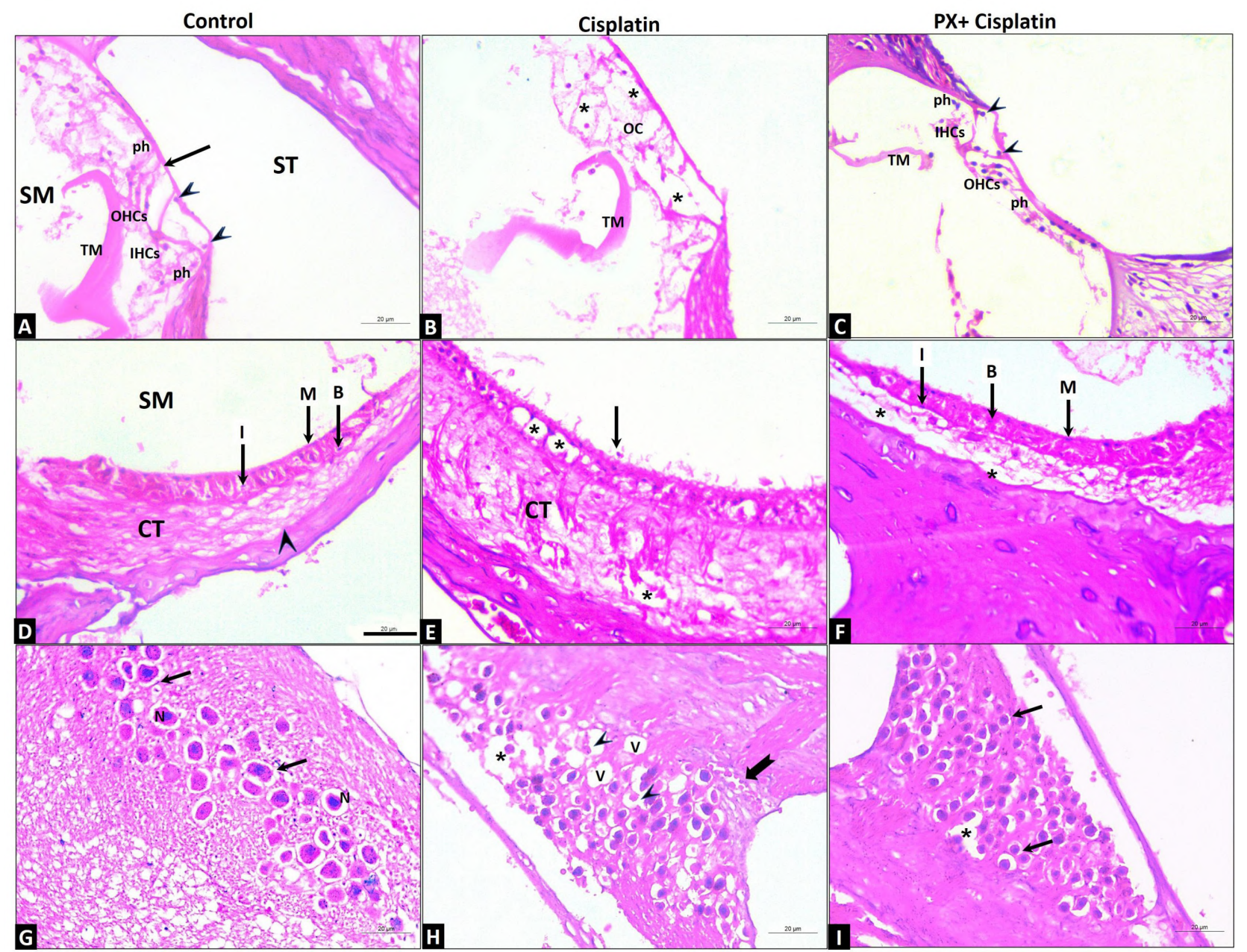

Fig. 3.- Photomicrographs of sections in the rat cochlea of different groups showing scala tympani (ST) and scala media (SM). The floor of the scala media (cochlear duct) is formed by basilar membrane (arrow), organ of Corti (OC) resting on this membrane. There are three rows of outer hair cells $(\mathrm{OHC})$ and one row of inner hair cells (IHC) with their supporting Phalangeal cells (ph) in the organ of Corti. Pillar cells (arrowhead) can be seen (A). Cisplatin group exhibits damaged organ of Corti (OC) along with vacuolation (asterisks) (B) while PTX+Cis group showing homogenous basilar membrane (arrow), outer hair cells (OHC) and inner hair cells (IHC). Other supporting cells were preserved, including phalangeal cells (ph) and pillar cells (arrowhead) (C). Stria vascularis epithelium of control group is seen resting on basal lamina (arrowhead). It consists of three layers of cells, namely, marginal (M), intermediate (I) and basal (B) cells. It is facing the endolymph of the Scala media (cochlear duct) (SM) and vascular connective tissue underlying (CT) (D). An ill-defined stria vascularis, denuded marginal cells (arrow) and vacuolation (asterisks) are seen in the Cis group, also apparent increase in thickness of the underlying connective tissue layer (CT) is observed (E). The stria vascularis marginal (M), intermediate (I), and basal layers (S) are intact, but the connective tissue layer (CT) has some vacuolation (asterisks) in the PTX+Cisplatin group (F). The spiral ganglion of the control group consists of large central nuclei in pseudo-unipolar neurons (N) and satellite cells(arrow) surrounding it (G). Cisplatin group spiral ganglion exhibits hemorrhage (notched arrow), nuclear degeneration with perinuclear halo (arrowhead), and vacuolation (asterisk) (H). These spiral ganglion neuron bodies are well identified as pseudo-unipolar neurons in Cisplatin group (I) but some vacuolation (asterisk) is still detected. H\&E, Scale bar $=20 \mu \mathrm{m}, 40 \mathrm{x}$. 


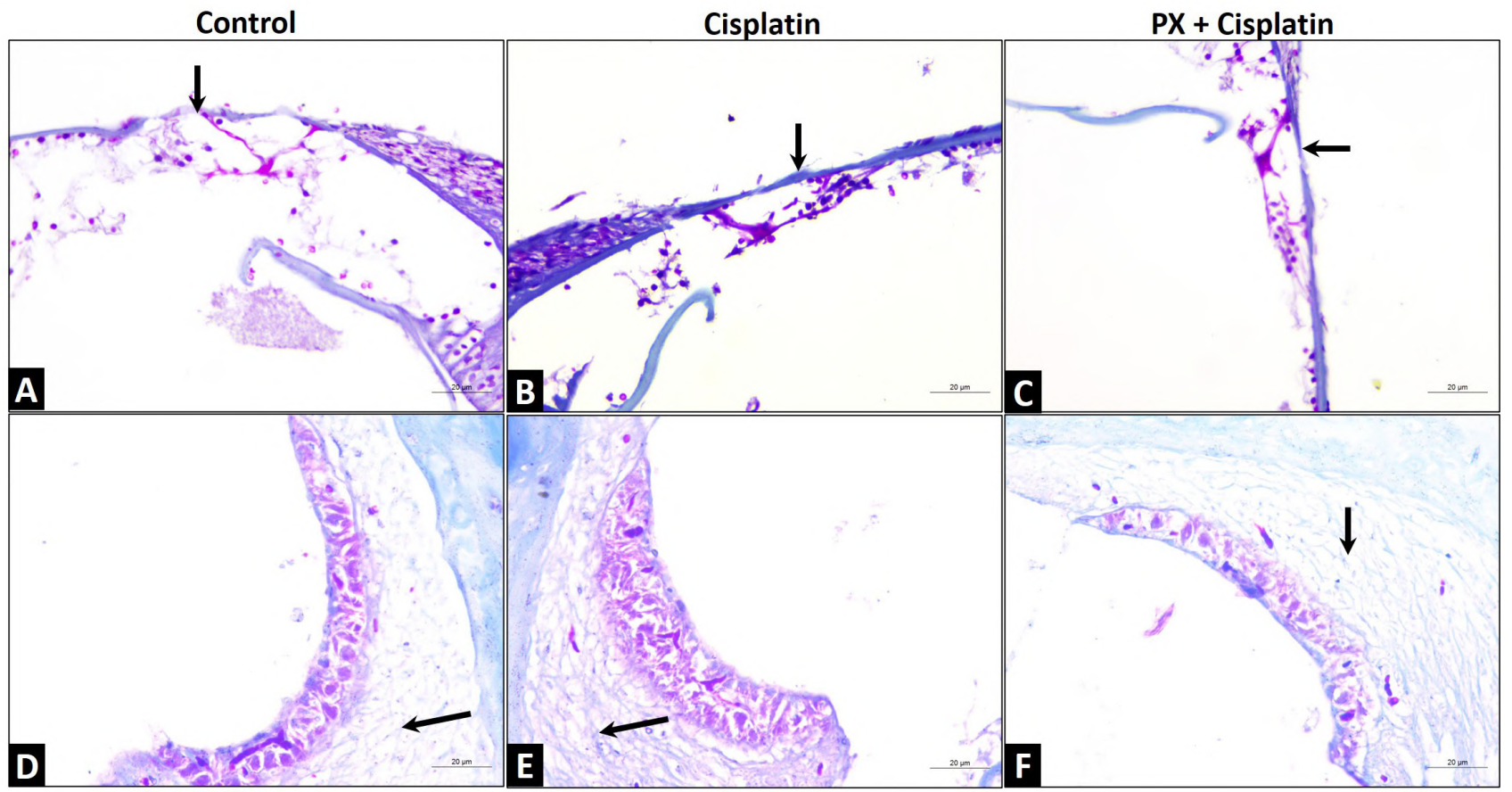

Fig. 4.- Photomicrographs of sections in the rat cochlea of different groups showing Control and PX+Cis groups have been deposited minimal collagen deposition in the basilar membrane (arrow) (A and C, respectively) and under the stria vascularis (D, F, respectively). In contrast, the Cis group exhibits an increased collagen deposit (B, E). Masson Trichrome, Scale bar $=20 \mu \mathrm{m}, 40 \mathrm{x})$.

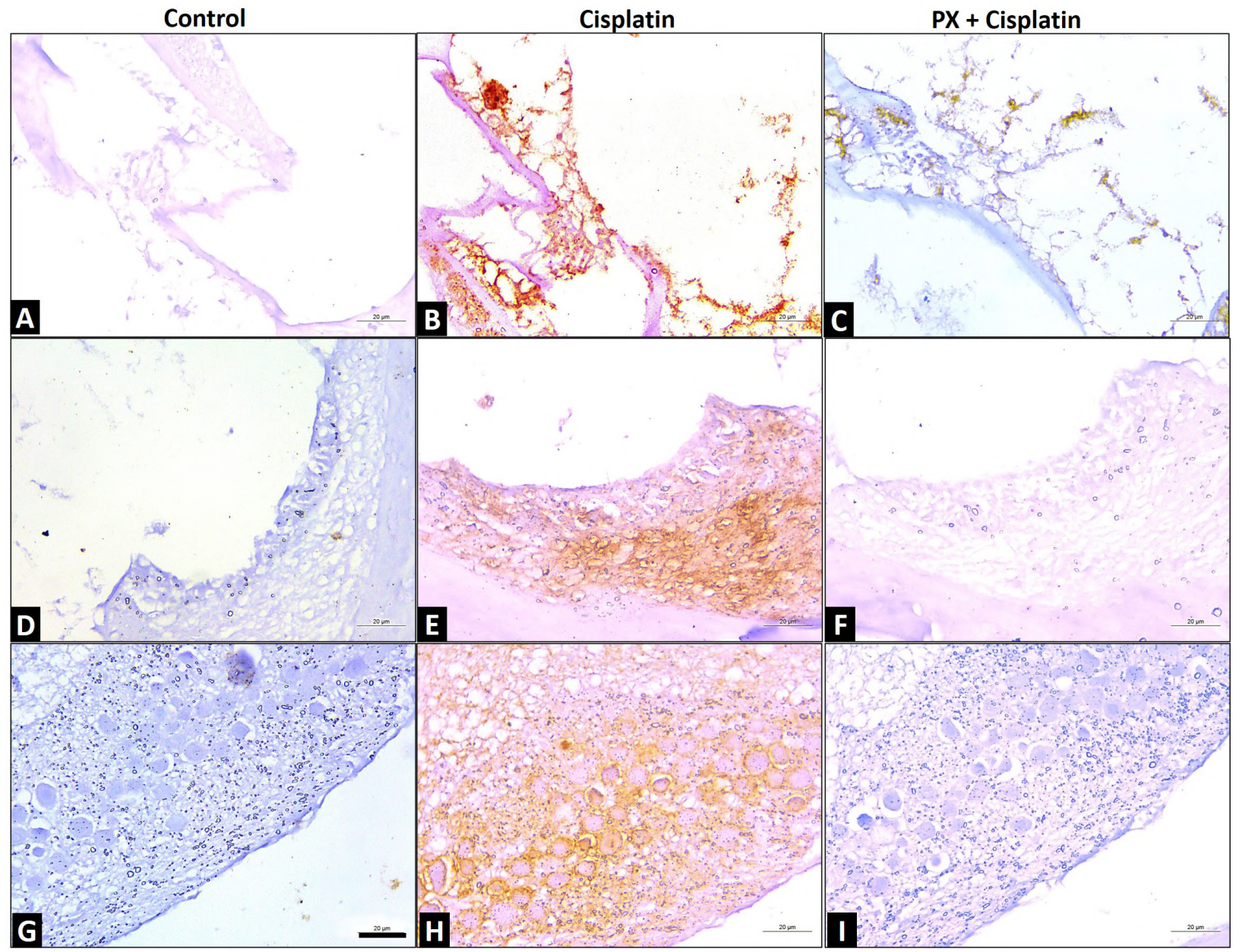

Fig. 5.- Photomicrographs of sections in the rat cochlea of different groups showing: A, D, G) A faint positive brown Caspases-3 reaction can be observed in the control group, organ of Corti, stria vascularis, and spiral ganglia. B, E, H) Cisplatin group exhibits marked expression in organ of Corti, stria vascularis and spiral ganglia. C, F, I) Caspase-3 reaction, however, is downregulated in the PX+Cis group. Caspase-3, Scale bar = $20 \mu \mathrm{m}, 40 \mathrm{x}$. 


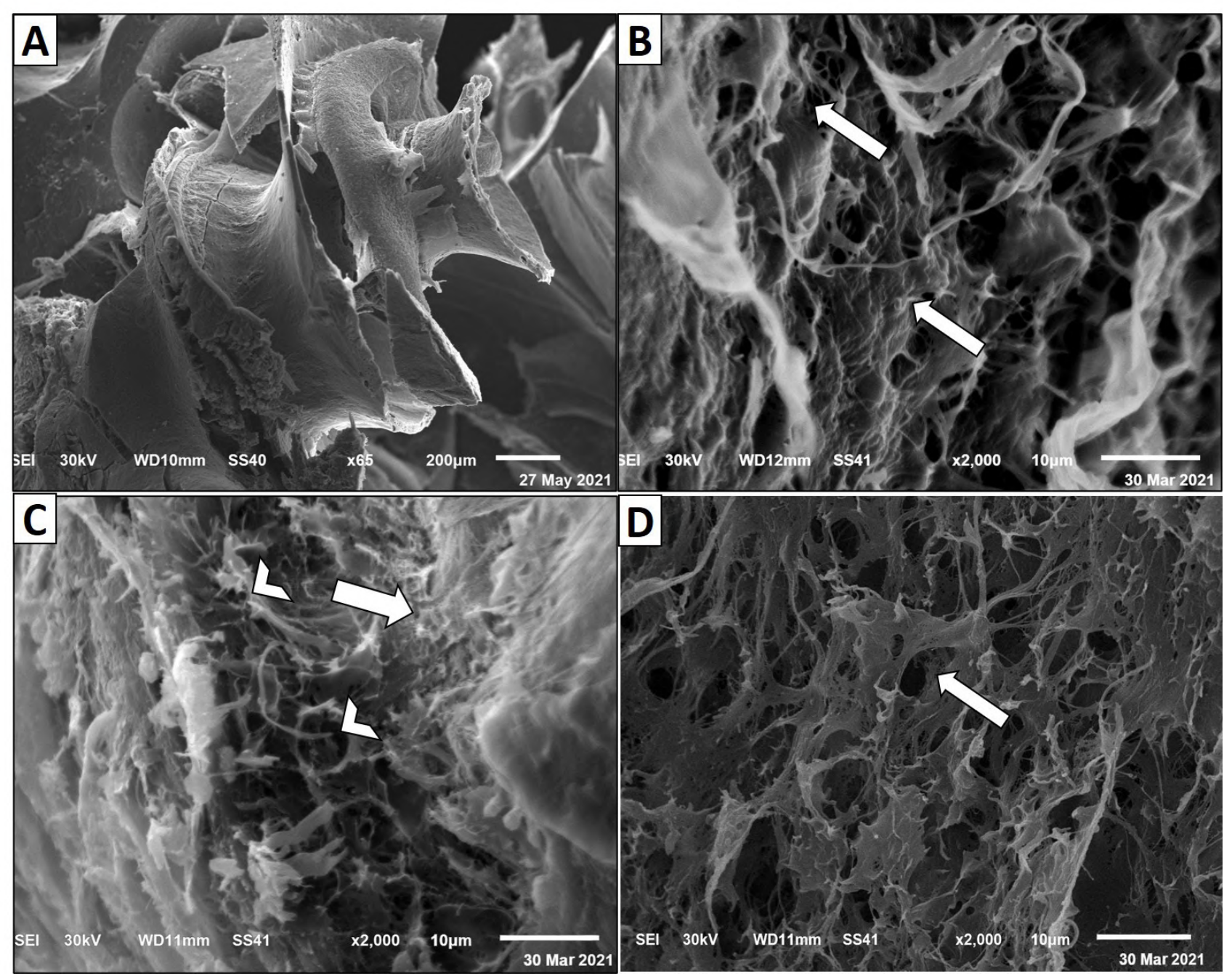

Fig. 6.- Scanning electron micrographs of rat cochlea of A, B) control group displays the spiral turn of the cochlea and cast of normal vasculature. C) Cisplatin group revealing a collapsed cast of cochlear vasculatures (arrow), disrupted veins and arteries can be seen (arrowhead). D) PX+Cis group normal cast of cochlear vasculatures (arrow). SEM, Scale bars: A = $200 \mu \mathrm{m}, \mathrm{x} 65$; B, C and D = $10 \mu \mathrm{m}, \mathrm{x} 2000$ ).

The cochlea of the PX+cisplatin group showed a normal cast of cochlear vasculatures (Fig. 6D). Cochlear hair cells were intact and arranged in a normal manner along with the normal architecture of stereocilia. However, areas of fusion of stereocilia of OHCs and cavitation were still detected (Fig. 7 G, H, I).

\section{DISCUSSION}

The antineoplastic drug cisplatin is widely used to treat a wide range of tumors. The most common side effects of this drug are nephrotoxicity, neurotoxicity, and ototoxicity. Ototoxicity is still cisplatin's most critical side-effect (RoldánFidalgo et al., 2016). Chemotherapy's powerful anti-tumor properties can be preserved by developing effective methods to reduce cisplatin ototoxicity (Soyalıç et al., 2016).
The results of our study clearly showed that pentoxifylline is protective against cisplatininduced ototoxicity. It was found that pentoxifylline prevented oxidative stress from occurring in the cochlea both biochemically and histologically after administration of cisplatin. Also, DPOAE findings demonstrated that pentoxifylline protected against cisplatin-induced hearing loss.

There is a lack of understanding of the molecular and cellular mechanisms of ototoxicity. Cisplatin damages the outer hair cells of the cochlea, which can lead to excessive free oxygen radical production in the organ of Corti, stria vascularis, spiral ligament and spiral ganglion cells (Yumusakhuylu et al., 2012; Lee et al., 2003). Moreover, cisplatin treatment is associated with decreased expression of endogenous antioxidants, 
thereby increasing oxidative damage. In addition, cisplatin can damage DNA and increase free radical production in the cochlea (Sagit et al., 2013).

The most prominent histopathological finding is hair cell degeneration. Furthermore, it results in the collapse of Reissner's membrane and atrophy of the support cells of the stria vascularis and the organ of Corti (Yumusakhuylu et al., 2012; Yazici et al., 2012).

In the current study, histopathological examination of the cochlea in the cisplatin group showed that the normal architecture of the organ of Corti was disrupted. All the hair cells, as well as their supporting cells, were fragmented and destroyed with a disrupted basement membrane, vacuolation and degeneration of stria vascularis marginal cells with an apparent increase in thickness and vacuolation of CT underneath stria vascularis. Similar findings have been reported by Soyalic et al. (2016), who demonstrated extreme histological damage to the organ of Corti in cisplatin-treated rats. Also, Akdemir et al. (2018) reported that cisplatin treatment reduced the number of outer hair cells in the organ of Corti of rats, and caused desquamation and erosion of vessels in the stria vascularis.

Although oxidative stress is thought to be the primary cause of this damage at the cellular level, the exact molecules and pathology responsible are unknown. Numerous studies have suggested that cisplatin can cause ototoxicity by causing an increase in free oxygen radicals within cellular structures in the cochlea. Furthermore, cisplatin has also been reported to reduce levels of antioxidant enzymes in the cochlea. In cochlear tissues, cisplatin causes the creation

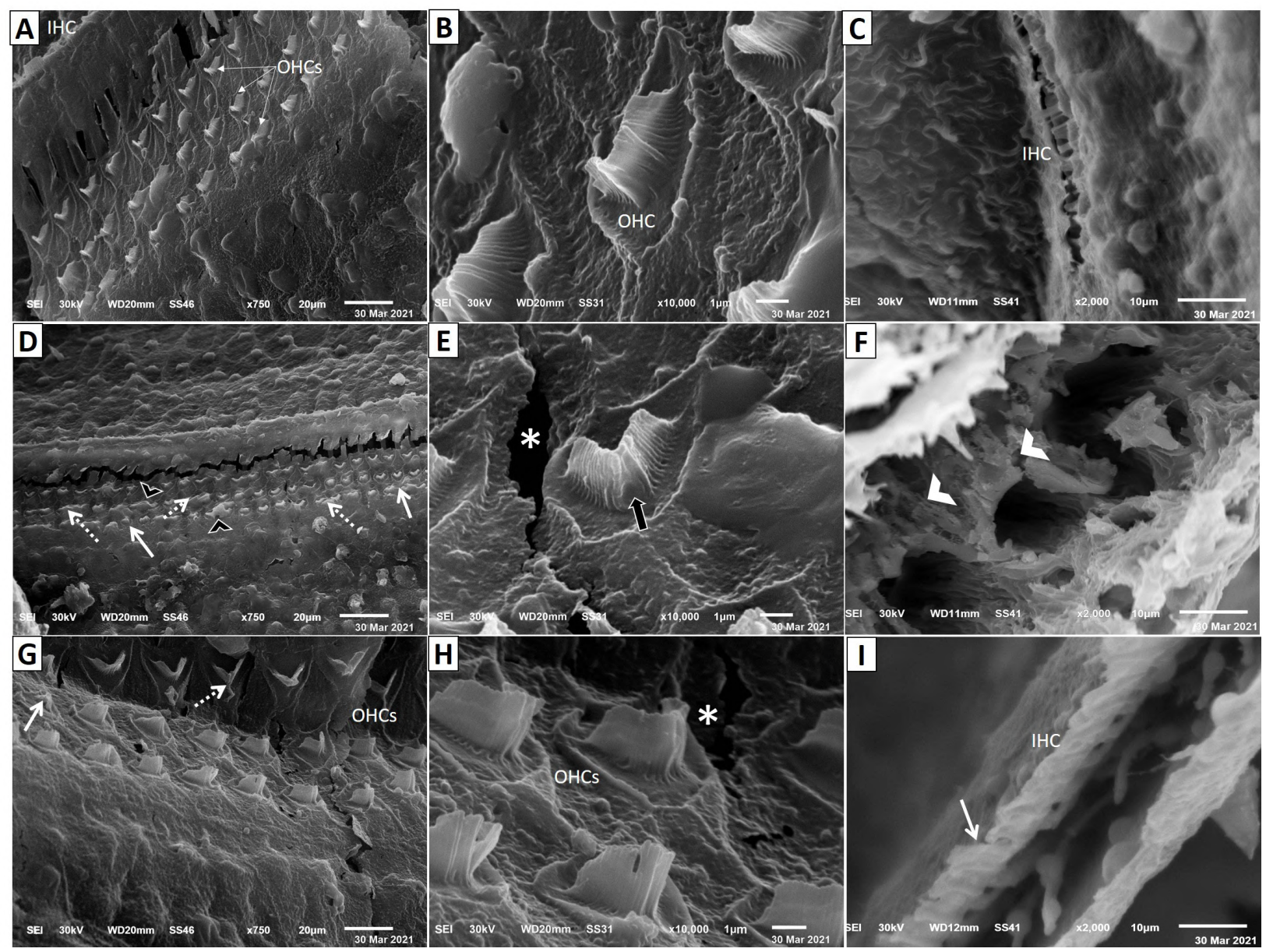

Fig. 7.- Scanning electron micrographs of rat cochlea of A, B, C) control group exhibits three rows of outer hair cells (OHCs) stereocilia and one row of inner hair cells (IHC) stereocilia. The regular arrangement of most of the stereocilia of the outer (OHCs) and inner hair cells (IHCs) is observed; Cis group (D, E, F) showing destructed (thin arrows), fused (dashed arrows), distorted (black arrowhead) and fragmented outer hair cells stereocilia (black arrow) and cavitation (asterisk). Loss of all stereocilia in the inner hair cells (white arrowheads). PX+Cis group (G, H, I) display intact stereocilia of both outer and inner hair cells (OHCs and IHC respectively) but areas destructed stereocilia (arrows) of OHC and IHC, fused stereocilia of OHCS (dashed

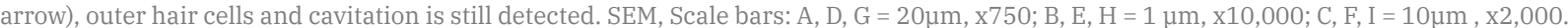


of dysfunctional proteins and enzymes by impairing DNA synthesis. This will increase free oxygen radicals and reduce the antioxidant enzyme system. Increased ROS and a reduced antioxidant system result in oxidative stress, a cytotoxic condition. Oxidative stress damages cochlear cells and causes cell death (Doğan et al., 2016).

The measurement of total antioxidant capacity (TAC) is a reasonable and accurate method to evaluate changes related to oxidative stress. By using the TAC, we can assess how various conditions affect oxidative statuses in vivo, such as ROS exposure and supplementation with galenic antioxidants. Additionally, plasma TAC values may provide information about antioxidant supplement absorption and bioavailability (Ghiselli et al., 2000).

The measurements of TAC in our study revealed that pentoxifylline wielded its effect on circulation. Levels of TAC did not differ between the control and cisplatin + pentoxifylline groups $(\mathrm{P}>$.05.), although the cisplatin group had significantly lower levels than the other groups. This was in agreement with Kinal et al. (2021), who found that cisplatintreated rats had significantly lower TAC value than controls. It has been shown that pentoxifylline could prevent cisplatin from decreasing the TAC level when administered with it.

As a result of the present study, the expression of Caspase-3 was significantly up-regulated in the cisplatin-treated rats' cochleae, confirming apoptotic changes. The results were consistent with the observation made by Kilic et al. (2019), who noticed high immunopositivity of Caspase-3 in the outer hair cells of cochleae of rats receiving cisplatin. Furthermore, Wang et al. (2004) found that cisplatin treatment activated caspase- 3 and caspase-9 in pigmented guinea-pig cochlear hair cells. In summary, Sheth et al. (2017) explained that caspases are involved in cisplatin-induced apoptosis of auditory cells, which is triggered by several upstream pathways. Inhibiting some or all of these pathways could be enough to rescue hair cells and hearing could be restored.

As is well known, drug toxicity mostly affects the outer hair cells in the organ of Corti. Normal otoacoustic emissions indicate healthy outer hair cells; however, diminished otoacoustic emissions reflect damaged hair cells. DPOAE and transient evoked otoacoustic emission (TEOAE) is used most commonly in clinical practice. Based on the studies conducted, DPOAE was shown to be more sensitive than TEOAE in detecting cisplatininduced hearing loss (American Academy of Audiology, 2009). Using DPOAE as an audiologist monitoring procedure for ototoxicity, we emphasized that a single dose of cisplatin (10 $\mathrm{mg} / \mathrm{kg}$ ) decreased the DPOAE amplitudes in rats. There have been similar findings reported by Kilic et al. (2019), De Araujo et al. (2019), Eryilmaz et al. (2016) and Soyalic et al. (2016). There is a wide range of possible frequencies that can be studied through DPOAE depending on the type of animal. Lopez-Gonzalez et al. (2000) identified them between 1 and $6 \mathrm{kHz}$ in Wistar rats; Sockalingan et al. (2000), between 2 and $8 \mathrm{kHz}$ for a signal/ noise ratio $\geq 3 \mathrm{~dB}$ SPL in albino rats.

The use of various approaches to treat cisplatin ototoxicity has increased over the past two decades. These include the administration of antioxidants or drugs that promote endogenous antioxidants. By activating the mitochondrial pathway, cisplatin induces apoptosis in hair cells. It is possible to prevent this by targeting the mitochondrial pathway. In addition, antiinflammatory agents can also be used in order to treat the pro-inflammatory mechanisms accompanying cisplatin therapy of the cochlea. Understanding the cisplatin entry process in hair cells and other cochlear cells might boost the development of drugs that prevent cisplatin from entering these cells without affecting the entrance of cisplatin into cancer cells (Sheth et al., 2017).

In light of these pathophysiologic processes, reduction of reactive oxygen species levels and an increase in antioxidant enzyme levels are thought to protect against ototoxicity caused by cisplatin. Numerous antioxidant agents have been studied for this purpose in ototoxicity. In our study, we explored the protective effect of PX, a powerful antioxidant agent, on cisplatin-induced ototoxicity.

The methylxanthine phosphodiesterase inhibitor PX exhibits powerful antioxidant, antiinflammatory and immune-regulatory effects 
(Shan et al., 2012). In the present work, the beneficial effect of PX against ototoxicity caused by cisplatin was examined based on histological, ultrastructural and immunohistochemical assessment. The coadministration of cisplatin with PX significantly ameliorated cochlear damage. It has been reported that PX can alleviate gentamicin effects (Stojiljkovic et al., 2009), cisplatin (Kim et al., 2003), glycerol (Akpolat et al., 2000) and cyclosporine-induced nephrotoxicity (Brunner et al., 1989). Moreover, Garcia et al. (2014) showed that PX significantly reduced blood glucose levels, fructosamine, HbA1c levels, and iNOS, COX-2, and TNF-alpha immunoreactivity in the diabetic pancreas, liver, and kidney tissue.

Ourresultsarealsoinlinewith thoseofStojiljkovic et al. (2009), who suggested that pentoxifylline might have renoprotective effects as a result of its vascular decongestant effects, and Ozer et al. (2009), who exhibited that tumor necrosis factoralpha (TNF- $\alpha$ ) and other cytokines are inhibited by pentoxifylline. Pentoxifylline has been demonstrated to protect against chemotherapyand amikacin-induced nephrotoxicity in several studies (Shifow et al., 2000). Pentoxifylline may provide otorenoprotection by inhibiting arachidonic acid metabolism and reducing pro-inflammatory mediators such as TNF- $\alpha$. In addition to activating macrophages, TNF- $\alpha$ also stimulates neutrophil proinflammatory secretion, causing apoptosis. This result in cell death, leading to necrosis of target organs. In addition, Aviado et al. (1984) stated that, in addition to scavenging free radicals, pentoxifylline also inhibits nitric oxide synthase.

\section{CONCLUSION}

As evidenced by our findings and results from DPOAE, biochemical findings, histological and ultrastructural analyses, PX significantly protects rats against cisplatin-induced ototoxicity. PX should be studied further in order to determine the appropriate dosages and indications to be used against cisplatin-induced ototoxicity before clinical use.

\section{REFERENCES}

AKDEMIR FNE, GOZELER MS, YILDIRIM S, ASKIN S, DORTBUDAK MB, KIZILTUNC A (2018) The effect of ferulic acid against cisplatininduced ototoxicity. Med-Science Int Medical J, 7(3): 528-531.

AKPOLAT T, AKPOLAT I, OZTURK H, SARIKAYA S, COŞAR A M, BEDIR A, KANDEMIR B (2000) Effect of vitamin E and pentoxifylline on glycerol-induced acute renal failure. Nephron, 84: 243-247.

AMERICAN ACADEMY OF AUDIOLOGY POSITION STATEMENT AND CLINICAL PRACTICE GUIDELINES (2009): Ototoxicity Monitoring. Reston, Virginia: American Academy of Audiology.

AMORA LDAS, MURASHIMA ADAB, ROSSATO M, MOREIRA MB, HYPPOLITO MA, FAGUNDES DJ (2013) The effects of hyperbaric oxygen therapy upon ototoxic injuries produced by amikacin in guinea pigs. Braz J Otorhinol, 79(3): 342-348.

AVIADO DM, PORTER JM (1984) Pentoxifylline: A new drug for the treatment of intermittent claudication. Mechanism of action, pharmacokinetics, clinical efficacy and adverse effects. Pharmacotherapy, 4(6): 297-307.

BRUNNER LJ, VADIEI K, IYER LV, LUKE DR (1989) Prevention of cyclosporine-induced nephrotoxicity with pentoxifylline. Ren Fail, 11: 97-104.

DE ARAUJO JG, SERRA LSM, LAUAND L, KÜCKELHAUS SAS, SAMPAIO ALL (2019) Protective effect of melatonin on cisplatininduced ototoxicity in rats. Anticancer Res, 39(5): 2453-2458.

DOĞAN M, POLAT H, YAŞAR M, KAYA A, BAYRAM A, ŞENEL F, ÖZCAN I (2016) Protective role of misoprostol against cisplatin-induced ototoxicity. Eur Arch Otorhinolaryngol, 273: 3685-3692.

EL-ANWAR MW, ABDELMONEM S, NADA E, GALHOOM D, ABDELSAMEEA AA (2018) Protective effect of pentoxifylline on amikacin-induced ototoxicity. Ear Nose Throat J, 97(8): E8-E12.

ERDEM T, BAYINDIR T, FILIZ A, IRAZ M, SELIMOGLU E (2012) The effect of resveratrol on the prevention of cisplatin ototoxicity. Eur Arch Otorhinolaryngol, 269: 2185-2188.

ERYILMAZ A, ELIYATKIN N, DEMIRCI B, BASAL Y, KURT OMURLU I, GUNEL C, BASAK S (2016) Protective effect of Pycnogenol on cisplatininduced ototoxicity in rats. Pharm Biol, 54(11): 2777-2781.

GARCIA FA, PINTO SF, CAVALCANTE AF, LUCETTI LT, MENEZES SM, FELIPE CF, ALVES AP, BRITO GA, CERQUEIRA GS, VIANA GS (2014) Pentoxifylline decreases glycemia levels and TNF-alpha, iNOS and COX-2 expressions in diabetic rat pancreas. Springerplus, 3: 283.

GHISELLI A, SERAFINI M, NATELLA F, SCACCINI C (2000) Total antioxidant capacity as a tool to assess redox status: critical view and experimental data. Free Radic Biol Med, 29(11): 1106-1114.

GILL SE, ROHAN M, MEHTA S (2015) Role of pulmonary microvascular endothelial cell apoptosis in murine sepsis-induced lung injury in vivo. Respir Res, 16: 109.

KALKANIS JG, WHITWORTH C, RYBAK LP (2004) Vitamin E reduces cisplatin ototoxicity. Laryngoscope, 114: 538-542.

KARA M, TÜRKÖN H, KARACA T, GÜÇLÜ O, UYSAL S, TÜRKYILMAZ M, DEMIRTAŞ S, DEREKÖY FS (2016) Evaluation of the protective effects of hesperetin against cisplatin- induced ototoxicity in a rat animal model. Int J Pediatr Otorhinolaryngol, 85:12-18.

KILIC K, SAKAT MS, AKDEMIR FNE, YILDIRIM S, SAGLAM YS, ASKIN S (2019) Protective effect of gallic acid against cisplatin-induced ototoxicity in rats. Braz J Otorhinolaryngol, 85(3): 267-274.

KIM YK, CHOI TR, KWON CH, KIM JH, WOO JS, JUNG JS (2003) Beneficial effect of pentoxifylline on cisplatin-induced acute renal failure in rabbits. Ren Fail, 25: 909-922.

KINAL ME, TATLIPINAR A, UZUN S, KESKIN S, TEKDEMIR E, OZBEYLI D, AKAKIN D (2021) Investigation of astaxanthin effect on cisplatin ototoxicity in rats by using otoacoustic emission, total antioxidant capacity, and histopathological methods. Ear Nose Throat J, 100(4): 198-205. 
KORACEVIC D, KORACEVIC G, DJORDJEVIC V, ANDREJEVIC S, COSIC $\mathrm{V}$ (2001) Method for the measurement of antioxidant activity in human fluids. J Clin Pathol, 54(5): 356-361.

LEE JE, NAKAGAWA T, KIM TS, IGUCHI F, ENDO T, DONG Y, YUKI K, NAITO Y, LEE SH, ITO J (2003) A novel model for rapid induction of apoptosis in spiral ganglions of mice. Laryngoscope, 113: 994-999.

LOPEZ-GONZALEZ MA, GUERRERO JM, ROJAS F, DELGADO F (2000) Ototoxicity caused by cisplatin ameliorated by melatonin and other antioxidants. J Pineal Res, 28: 73-80.

NASIRI-TOOSI Z, DASHTI-KHAVIDAKI S, KHALILI H, LESSANPEZESHKI M (2013) A review of the potential protective effects of pentoxifylline against drug-induced nephrotoxicity. Eur J Clin Pharmacol, 69:1057-1073.

OZER MK, ASCI H, ONCU M, YESILOT S, SAVRAN M, BAYRAM D, CICEK E (2009) Effects of pentoxifylline on amikacin-induced nephrotoxicity in rats. Ren Fail, 31(2): 134-149.

ROLDAN-FIDALGO A, MARTÍN SALDANA S, TRINIDAD A, OLMEDILLA-ALONSO B, RODRÍGUEZ-VALIENTE A, GARCÍA-BERROCAL JR, RAMÍREZ-CAMACHO R (2016) In vitro and in vivo effects of lutein against cisplatin-induced ototoxicity. Exp Toxicol Pathol, 68: 197-204.

RYBAK LP (2007) Mechanisms of cisplatin ototoxicity and progress in otoprotection. Curr Opin Otolaryngol Head Neck Surg, 15: 364-369.

SAGIT M, KORKMAZ F, AKCADAG A, SOMDAS MA (2013) Protective effect of thymoquinone against cisplatin-induced ototoxicity. Eur Arch Otorhinolaryngol, 270: 2231-2237.

SALEHI P, AKINPELU OV, WAISSBLUTH S, PELEVA E, MEEHAN B, RAK J, DANIEL SJ (2014) Attenuation of cisplatin ototoxicity by otoprotective effects of nanoencapsulated curcumin and dexamethasone in a Guinea pig model. Otol Neurotol, 35: 1131-1139.

SHAN D, WU HM, YUAN QY, LI J, ZHOU RL, LIU GJ (2012) Pentoxifylline for diabetic kidney disease. Cochrane Database Syst Rev, 2: CD006800.

SHETH S, MUKHERJEA D, RYBAK LP, RAMKUMAR V (2017) Mechanisms of cisplatin-induced ototoxicity and otoprotection. Front Cell Neurosci, 11: 338.

SHIFOW AA, NAIDU MU, KUMAR KV, PRAYAG A, RATNAKAR K S (2000) Effect of pentoxifylline on cyclosporine-induced nephrotoxicity in rats. Indian J Exp Biol, 38(4): 347-352.

SOCKALINGAM R, FREEMAN S, CHERNY L, SOHMER H (2000) Effect of high-dose cisplatin on auditory brainstem responses and otoacoustic emissions in laboratory animals. Am J Otol, 21: 521-527.

SOYALIÇ H, GEVREK F, KOÇ S, AVCU M, METIN M, ALADAG I (2016) Intraperitoneal curcumin and vitamin $\mathrm{E}$ combination for the treatment of cisplatin-induced ototoxicity in rats. Int J Pediatr Otorhinolaryngol, 89: 173-178.

STOJILJKOVIC N, VELJKOVIC S, MIHAILOVIC D, STOILJKOVIC M, RADENKOVIC M, RANKOVIC G, RANDJELOVIC P (2009) Protective effects of pentoxifylline treatment on gentamicin-induced nephrotoxicity in rats. Ren Fail, 31:54-61.

STRUCK MB, ANDRUTIS KA, RAMIREZ HE, BATTLES AH (2011) Effect of a short-term fast on ketamine-xylazine anesthesia in rats. $J \mathrm{Am}$ Assoc Lab Anim Sci, 50(3): 344-348.

SUVARNA SK, BANCROFT JD, LAYTON C (2012) Bancroft's Theory and Practice of Histological Techniques, $7^{\text {th }}$ ed. Churchill Livingstone Elsevier, Philadelphia, PA.

WANG GG, LU XH, LI W, ZHANG C (2011) Protective effects of luteolin on diabetic nephropathy in STZ-induced diabetic rats. Evid based Complement Alternat Med, 2011: 323171.

WANG J, LADRECH S, PUJOL R, BRABET P, WATER VD, T R, PUEL JL (2004) Caspase inhibitors, but not c-Jun NH2-terminal kinase inhibitor treatment, prevent cisplatin-induced hearing loss. Cancer Res, 64: 92179224.
XIE J, TALASKA AE, SCHACHT J (2011) New developments in aminoglycoside therapy and ototoxicity. Hear Res, 281: 28-37.

YAZICI ZM, MERIC A, MIDI A, ARINC YV, KAHYA V, HAFIZ G (2012) Reduction of cisplatin ototoxicity in rats by oral administration of pomegranate extract. Eur Arch Otorhinolaryngol, 269: 45-52.

YUMUSAKHUYLU AC, YAZICI M, SARI M, BINNETOGLU A, KOSEMIHAL E, AKDAS F, SIRVANCI S, YUKSEL M, UNERI C, TUTKUN A (2012) Protective role of resveratrol against cisplatin induced ototoxicity in Guinea pigs. Int J Pediatr Otorhinolaryngol, 76: 404-408. 